\title{
Sexual Intensity of Adolescents' Online Self-Presentations: Joint Contribution of Identity, Media Consumption, and Extraversion
}

Piotr S. Bobkowski, University of Kansas

Autumn Shafer, University of Oregon

Rebecca R. Ortiz, Texas Tech University

Submitted for publication in Computers and Human Behavior

\begin{abstract}
Adolescents produce and distribute a vast quantity of digital media content. A growing literature examines the sexually explicit (i.e., nude) content that adolescents share online. Because adolescents' sexual content need not be sexually explicit, however, this study examined the sexual intensity with which adolescents choose to present themselves in the context of a social media platform. Exemplifying the variability of adolescents' online sexual self-presentations, survey participants $(N=265$; age range: $13-15$ years $)$ constructed social media profiles using components (e.g., photos, fashion brands) that varied in sexual intensity. In accord with predictions drawn from the Media Practice Model, the study found that the sexual intensity of adolescents' online self-presentations is a product of the sexual self-concept, a relationship that is partially mediated by sexual media diet and moderated by extraversion. This study bridges emerging research on sexual self-presentation with established literature on adolescents' sexual media uses and effects.
\end{abstract}

Keywords: adolescents; mass media; self-concept; sexting; sexual media diet; social media 


\section{Introduction}

Adolescents in the United States produce and distribute a vast quantity of digital media content (Madden, Lenhart, Cortesi, \& Gasser, 2013). Researchers working at the intersection of media use and adolescent sexual development are increasingly interested in examining the sexual media that adolescents produce and distribute, and the implications of these behaviors for their sexual health and identity formation. "Sexting" (i.e., sending sexually explicit photos or messages via an electronic device) has received the most research attention among the sexual media production practices in which adolescents engage (e.g., Baumgartner, Sumter, Peter, Valkenburg, \& Livingstone, 2014; Dake, Price, Mazriaz, \& Ward, 2012; Klettke, Hallford, \& Mellor, 2014; Mitchell, Finkelhor, Jones, \& Wolak, 2012; Temple \& Choi, 2014; Temple, Paul, Berg, Le, McElhany, \& Temple, 2012). Adolescents can produce and distribute sexual text, photo, video and audio content via many social media platforms (e.g., Facebook, Instagram, YouTube, Snapchat). Some studies have focused specifically on young people's sexual disclosures in these channels (e.g., Bobkowski, Brown, \& Neffa, 2012; Ringrose, 2011).

The present study advances two conceptual components within the literature on adolescents' sexual media production and distribution. Much of the prior work in this domain assessed behaviors related to sexually explicit, that is, "nude or nearly nude" (Mitchell et al., 2012, p. 15) digital images or videos. Young people, however, also can present themselves online sexually without being sexually explicit (Smahel \& Subrahmanyam, 2014). They can communicate a level of sexual suggestiveness through physical poses, facial expressions, and clothing in their online photos, for instance, or through the music or brands they endorse (i.e., "Like") on social media.

This study therefore first widens the analytic lens by examining the sexual intensity with which adolescents present themselves in digital spaces, including subtle and suggestive sexual 
content. Second, the study conceptualizes and tests how these online sexual self-presentations are shaped by the adolescents' own sexual identities, the sexual media they report consuming, and their personality. Prior studies have identified several discrete correlates of adolescents' digital sexual self-presentations including demographics, personality traits, and overall media use (Baumgartner et al., 2014; Mitchell et al., 2012). This study advances this literature by using a theoretical framework informed by the Media Practice Model (MPM) (Shafer, Bobkowski, \& Brown, 2013; Steele \& Brown, 1995) to integrate components of both identity, sexual media consumption, and personality as mutual contributors to adolescents' sexual self-presentations.

Data from an online questionnaire completed by 26513 - to 15 -year-olds were used to test the study's theoretical framework with a moderated mediation model (Hayes, 2013). Exemplifying the variability of adolescents' online sexual self-presentations, participants selected elements that varied in sexual intensity to represent themselves in a fictitious social media platform. This study contributes to the growing literature and theory on adolescents' sexual self-presentation by bridging this emerging research domain to the established literature on adolescents' sexual media uses and effects (e.g., Wright, 2011). There are also practical advantages of the more comprehensive understanding of the relationships between identity, sexual media consumption, personality, and sexual self-presentation in digital spaces that this study advances. Most teens growing up today will make decisions about how to present themselves in social media. For instance, $91 \%$ of teens who use social media post photos of themselves, and $84 \%$ post about their media interests (Madden et al., 2013). A more thorough understanding of the factors involved in the sexual nature of adolescents' social media selfpresentations can inform related policies and educational initiatives on such topics as online privacy, online safety, and sexual health. 


\section{Conceptual Background}

\subsection{Adolescents' Sexual Media Distribution and Identity}

The vast majority of adolescents in the United States have regular access to the Internet, and most own at least one personal mobile device, such as a smartphone and/or a tablet (Madden, Lenhart, Duggan, Cortesti, \& Gasser, 2013). Most adolescents are active on at least one social media platform (e.g., Facebook, Instagram), and many are active on multiple platforms (Madden, Lenhart, Cortesti, et al., 2013; Madden, 2013). Using their mobile devices and social media platforms, adolescents share with their social networks the digital content they create or encounter online (i.e., text, photos, videos). Some of this content can be sexual in nature. Approximately one in 10 adolescents $(10.2 \%)$ has presented him- or herself sexually by distributing sexually suggestive text or photo content, according to a meta-analysis of six studies (Klettke et al., 2012).

Studies of adolescents' sexual self-presentations in digital spaces-specifically, sexting - have linked the incidence and frequency of these behaviors with a series of discrete demographic and behavioral correlates. Age predicts sexting, for example, with older adolescents more likely to sext than younger adolescents (Baumgartner et al., 2014; Dake et al., 2012). Some evidence also suggests that non-white adolescents are more likely to sext than their white peers (Dake et al., 2012). Adolescents who are sexually active are more likely to sext than those who are not sexually active; and adolescents who engage in risky sexual behaviors are more likely to sext than those who do not (Dake et al., 2012; Temple et al., 2012). Greater Internet use and more frequent texting also are linked to a higher probability of sexting (Baumgartner et al., 2014; Dake et al., 2012). While these prior studies identified discrete populations and behaviors associated with higher incidence of sexual self-presentation through sexting, integrative research that examines how combinations of these characteristics relate to adolescents' sexual self- 
presentations is lacking. A more cohesive understanding of how these factors combine to inspire adolescents' sexual presentations can inform educational initiatives and policies related to online safety, online privacy, and sexual health.

This study aims to frame sexual self-presentation online within a coherent theoretical context and to conceptualize it as the outcome of identity, media consumption, and personality. According to the Media Practice Model, the production and distribution of self-expressive digital content constitutes one component of a broader, self-reinforcing process (Shafer et al., 2013). In the model's original conceptualization, Steele and Brown (1995) found through interviews with adolescents in their bedrooms that most forms of media in which the adolescents engaged had connections to how they made sense of their lives, such that their own identities influenced what media they interacted with and how they interpreted and applied these media to their lives. The model (see Figure 1) thus holds that adolescents' identities shape their media selections, interactions, and applications, and that these practices in turn also influence the adolescents' identities. Adolescents' backgrounds and interests, which reflect their identities and "lived experiences," play a role in how they select, interpret, and apply media and eventually, in a cyclical fashion, how they see themselves (i.e. their self-concepts) in both private and public spaces (Hawk, Vanwesenbeeck, de Graaf, \& Bakker, 2006; Peter \& Valkenburg, 2006; Steele, 1999; Ward, 2003). Peter and Valkenburg (2006), for example, found that adolescents' exposure to sexually explicit materials was predicted by identity indicators, such as gender and sexual interest, and by selection and exposure to other sexual media. Steele (1999) found that how teens saw themselves (i.e., their identities) influenced the type of media they liked best, how they interacted with that media, and thus how they applied that media to their everyday lives. 


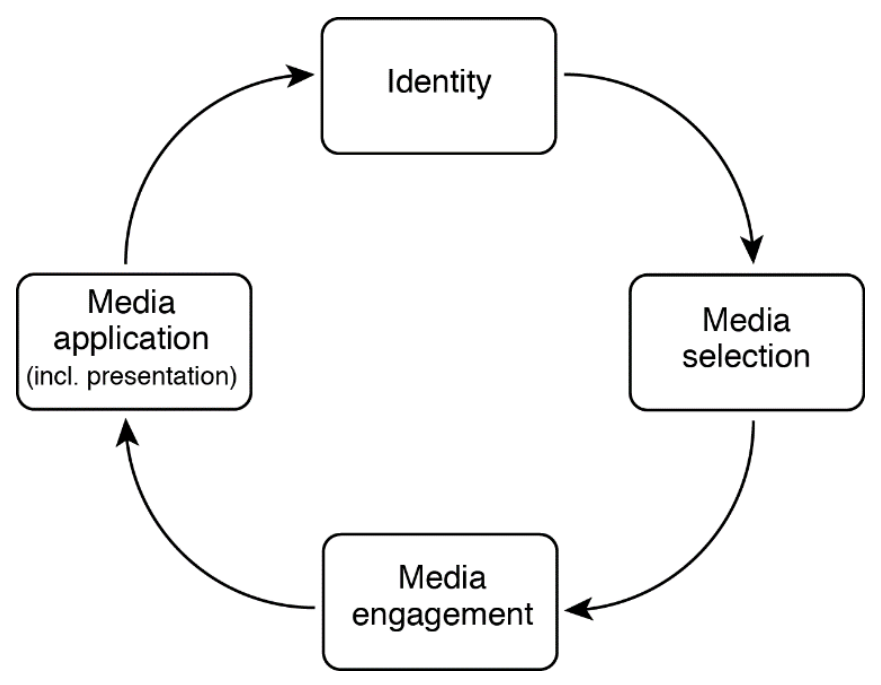

Figure 1

The Media Practice Model, showing the cyclical process by which an adolescent's identity shapes his or her media selection, interaction, and application; these practices, in turn, also influence the adolescent's identity.

Similarly, according to the Media Practice Model, whether an adolescent distributes a piece of media depends on the extent to which the media reflect the adolescent's identity (Shafer et al., 2013). Adolescents can "Like," "Share," "Retweet," and "Favorite" media content in social media platforms like Facebook, Instagram, Twitter, and Tumblr, and disseminate these self-referencing media to their social networks. They can also create and distribute social media updates, selfies, digital videos, and other forms of digital self-presentation. In creating and sharing all of this content, adolescents draw on their identities and the media they consume, producing media that reflect their real and aspirational selves (Shafer et al., 2013) and coconstructing their identities in the digital realm (Smahel \& Subrahmanyam, 2014).

Adolescents' use and production of sexual media thus relates to the sexual components of their identities, that is, for the purpose of this study, their sexual self-concepts (Breakwell \& 
Millward, 1997; Impett \& Tolman, 2006; Rostosky, Dekhtyar, Cupp, \& Anderman, 2008;

Winter, 1988). The sexual self-concept is often defined as how an individual perceives him or herself as a sexual person, such as how confident they feel or the level of agency and control they perceive having in sexual situations. These self-perceptions are often related to an individual's sexual behaviors or attitudes, derived from previous experiences and motivations (Snell, Fisher, \& Schuh, 1992; Snell, 1998). College students score higher than high school students on measures of sexual self-concept, and older college students score higher than younger college students (Winter, 1988). Higher sexual self-concept scores also correlate with greater sexual experience, higher sexual satisfaction (Impett \& Tolman, 2006), and increased likelihood of contraceptive use (Winter, 1988), but not with earlier sexual debut or the number of sexual partners (Impett \& Tolman, 2006). Although studies link correlates of the sexual selfconcept, like sexual experience and sexual risk-taking, with forms of sexual self-presentation (e.g., Dake et al., 2012; Temple \& Choi, 2014), research has not examined explicitly the relationship between the sexual self-concept and the intensity of sexual self-presentation. We propose that the sexual self-concept is related to the extent to which young people present themselves sexually in online spaces.

\subsection{The Role of Sexual Self-Concept in Sexual Self-Presentation}

Individuals generally use digital messaging and social media services to augment their offline relationships, with close friends being the leading recipients of digital self-disclosures (Desjarlais \& Willoughby 2010; Jones et al., 2013; Reich, Subrahmanyam, \& Espinoza, 2012). Because of this correspondence between online users' offline and online social networks, how individuals present themselves online tends to match their offline identities and behaviors. Individuals who are more prosocial in offline relationships, for example, also tend to be more prosocial in social media (Wright \& Li, 2011), while those who readily share product 
information offline also are more likely to share product information in digital venues (Ho \& Dempsey, 2010).

Given the high correspondence between social media users' online and offline communication behaviors, there is reason to expect that the intensity of adolescents' sexual selfpresentations will align with their offline sexual identities, that is, their sexual self-concepts. Studies on sexting, for example, show that among teens, sexting is often part of romantic relationships (Mitchell et al., 2012). College students similarly are more likely to sext if they are in a romantic relationship than when they are single (Delevi \& Weisskirch; Dir, Coskunpina, Steiner, \& Cyders, 2013), and sexting is more likely to occur between partners in committed sexual relationships than between casual sexual partners (Drouin, Vogel, Surbey, \& Stills, 2013). Correlates of the sexual self-concept, such as sexual activity, relate to sexting incidence. Individuals who are sexually active are more likely to sext than those who are not sexually active (Dake et al., 2012; Dir et al., 2013; Gordon-Messer, Bauermeister, Grodzinski, \& Zimmerman, 2013; Rice et al., 2012; Temple et al., 2012). In line with these findings on sexting incidence, we expect that the intensity with which adolescents communicate their sexual selves online relates to who they understand themselves to be sexually, that is, their reported sexual self-concepts. Accordingly, we predict that:

H1: There will be a positive association between the sexual self-concept and the sexual intensity of online self-presentations: adolescents with stronger sexual self-concepts will tend to self-present with greater sexual intensity; those with weaker sexual self-concepts will tend to self-present with less sexual intensity.

\subsection{The Mediating Role of the Sexual Media Diet}

Whereas prior studies examined online sexual communication (e.g., sexting) independent of sexual media consumption, the Media Practice Model suggests that adolescents' sexual media 
diets - the sexual messages they consume in media such as music videos, magazines, and TV programs - mediate the link between their sexual self-concepts and the sexual intensity of their self-presentations (Shafer et al., 2013). Adolescents' sexual media diets constitute the totality of the sexual content to which adolescents expose themselves (Brown et al., 2006; Pardun, L'Engle, \& Brown, 2005). To conceptualize sexual media diet as a mediator, it is important first to assert that adolescents' sexual self-concepts shape their sexual media diets.

Whether or not adolescents view or hear sexual media and if so, how much, can depend on the timing of their pubertal development and on influences from their families, peers, religious groups, and other social stimuli (Shafer et al., 2013). For instance, adolescents who feel less restrained about sex and those who express greater preoccupation with sex are more likely to regularly view online pornography than those who are not as permissive or interested in sex (Doornwaard, van den Eijinden, Overbeek, ter Bogt, 2015). At different points in their sexual development, adolescents may seek sexual information or guidance from the media (Bleakley, Hennessy, \& Fishbein, 2011), akin to news consumers' selective exposure to news that is relevant to their lives (Knobloch, Carpentier, \& Zillmann, 2003; Carpentier, 2008). Easily accessible online pornography, for instance, holds instructional value for some teens, as evidenced by adolescents enacting sexual behaviors they learn from online pornography (Rothman, Kaczmarsky, Jansen, \& Baughman, 2014).

In all, adolescents' sexual self-concepts- how they view themselves as sexual beingsshape their sexual media diets. We thus predict that:

H2: There will be a positive relationship between the sexual self-concept and the sexual media diet: adolescents with stronger sexual self-concepts will tend to have more sexual media diets; those with weaker sexual self-concepts will tend to have less sexual media diets. 
The mediating position of the sexual media diet means that while it is shaped by the sexual self-concept, it also informs adolescents' sexual self-presentation. According to the Media Practice Model, adolescents apply elements of media content to their identities (Shafer et al., 2013). In the 20th century, this manifested exclusively in "offline" activities, such as displaying celebrity posters on bedroom walls or assuming celebrities' fashions (Steele \& Brown, 1995). Such displays signaled to the adolescents themselves and to those around the adolescents, their appropriation of identity elements from the media. Youth today have many other means through which to signal the application of the media to elements of their identities, such as online and other digital spaces. Easy access to various digital recording, editing, and distribution methods makes the application of media content more immediate and more public than in previous decades (Shafer et al., 2013).

Although research thus far has not framed sexting and other online sexual communication practices as being the 21 st-century version of applying sexual content from the media to communicate one's identity, analogous research in the political domain shows that the consumption of political news predicts both greater political disclosure online (Macafee, 2013; Valenzuela, Kim, \& Gil de Zúñiga, 2012) and more conventional political disclosures, such as displaying campaign buttons and bumper stickers (Shah \& Scheufele, 2006). Studies also illustrate that the sexual media that adolescents create and share likely reflect the symbols, language, scripts, and tone of the sexual media they consume. College students who use online pornography are more likely to electronically send seminude or nude photos or videos of themselves than those who do not watch pornography (Crimmins \& Seigfried-Spellar, 2014). Adolescent females draw on sexual scripts from hip hop music to present themselves sexually in their online home pages as virgins, freaks, down-ass chicks, and pimpettes (Stokes, 2007). Adolescent males, meanwhile, use their phones to record themselves performing sexual acts in 
the style of amateur pornography they consume online and share with their friends (Rothman et al., 2014).

In all, the consumption of sexual media seems to be a necessary antecedent to adolescents' production and dissemination of sexual media. We thus expect that increased consumption of sexual media — a greater sexual media diet — is a precursor for presenting oneself more sexually in online platforms.

H3: There will be a positive association between the sexual media diet and the sexual intensity of online self-presentations: adolescents with more sexual media diets will tend to self-present with greater sexual intensity; those with less sexual media diets will tend to self-present with less sexual intensity.

Given $\mathrm{H} 2$ and $\mathrm{H} 3$, we also predict that,

H4: Sexual media diet will mediate the relationship between the sexual self-concept and the sexual intensity of online self-presentations.

\subsection{The Moderating Role of Extraversion}

Personality traits also may contribute to how sexually adolescents present themselves in digital spaces (Michikyan, Subrahmanyam, \& Dennis, 2014). Personality traits constitute individuals' dispositions to think, feel, and behave in somewhat consistent ways across unique situations (McCrae \& John, 1992). Extraversion, a dimension of the universal five-factor model of personality (McCrae \& John, 1992), is associated with expression-oriented behaviors both offline and online. Higher extraversion distinguishes individuals who experience more positive affect, are more outgoing, energetic, and assertive, and who participate in more social activities than introverts, that is, those who are lower in extraversion (Wilt \& Revelle, 2009).

Extraverts generally engage in more self-disclosure (Archer, 1979; Cozby, 1973) and behave differently than introverts in digital spaces. Extraverts are more likely than introverts to 
self-disclose using online messaging services (Peter, Valkenburg, \& Schouten, 2005), to use social media like Facebook (Ryan \& Xenos, 2011), and to connect with more friends via social media (Amichai, Hamburger, \& Vinitzky, 2010). Overall, extraverts engage in more selfdisclosure on Facebook than introverts: they upload more photos, post more status updates and comments, and click "Like" and "Share" more frequently (Lee, Ahn, \& Kim, 2014). With regard to digital sexual communication, extraverts are more likely than introverts to send sexual text messages and to send digital sexual propositions (Delevi \& Weisskirch, 2013).

In the context of the intensity of sexual self-disclosure, given extraverts' general tendency to disclose more than introverts in social media, we predict that extraversion will elevate the intensity of adolescents' sexual self-presentations beyond the direct influence of the sexual self-concept and sexual media diet. Accordingly, we predict that:

H5: Extraversion will moderate positively the relationship between the sexual self-concept and the sexual intensity of online self-presentations.

H6: Extraversion will moderate positively the relationship between sexual media diet and the sexual intensity of online self-presentations.

Together, our conceptualization offers a test of the Media Practice Model (Shafer et al., 2013), in that we propose that adolescent identity (sexual self-concept) influences media selection (sexual media diet), and that while moderated by personality (extraversion), these factors influence how adolescents use media content to present themselves in digital spaces (sexual intensity of online self-presentation). Figure 2 illustrates the proposed moderated mediation model of sexual intensity constructed from the six hypotheses. 


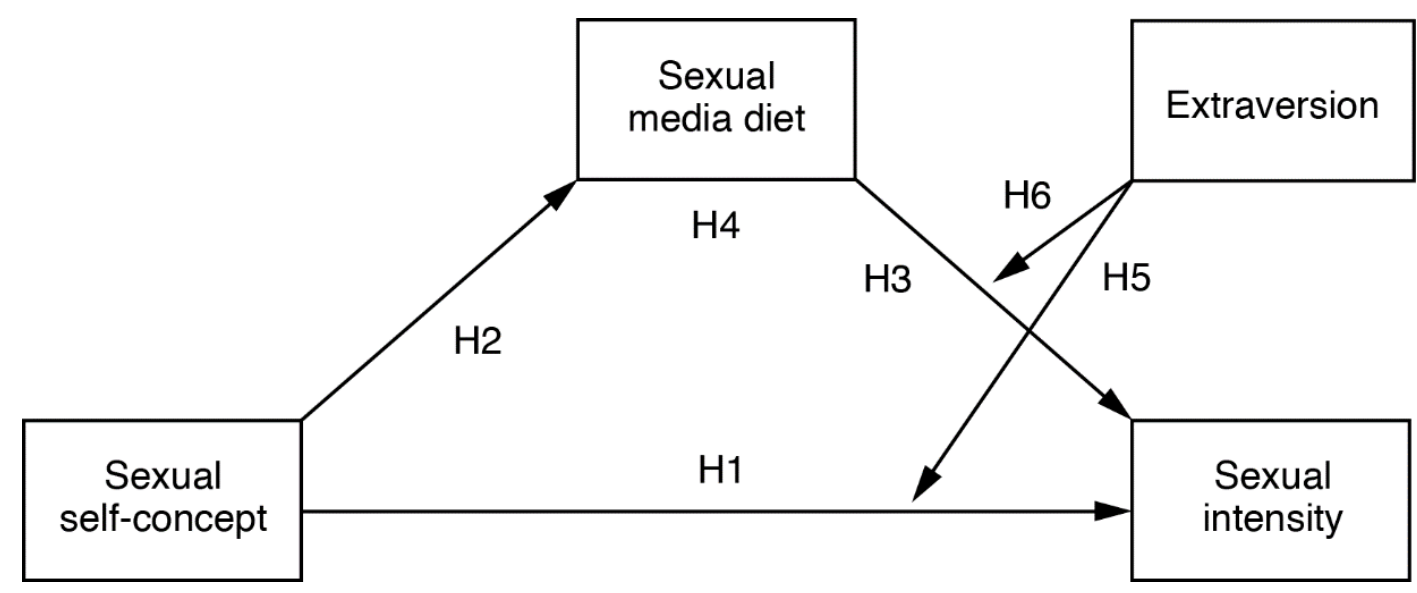

Figure 2

Proposed moderated mediation model predicting sexual intensity from sexual self-concept, sexual media diet and extraversion.

\section{Methods}

\subsection{Participants}

\subsubsection{Demographics}

The proposed conceptual model was tested with data from an online questionnaire that was delivered via Qualtrics, an online survey service. The respondent panel consisted of 265 participants. Slightly more adolescent females participated (55.09\%) than males. Almost half were 14 years old (45.28\%; 33.21\% were 13 years old; $21.51 \%$ were 15 years old), and majority identified as "white or Caucasian" (69.43\%; $15.47 \%$ as "black or African American;" 10.19\% as “Latino(a) or Hispanic;” 3.77\% as “Asian, Asian American, or Pacific Islander;” .75\% as “American Indian or Alaska Native;" and $.38 \%$ as “Other”). To include adolescent participants from both lower and higher socioeconomic backgrounds, quotas were imposed during participant recruitment so that the final panel would be almost evenly split between adolescent respondents who were eligible for free lunch (33.96\%), reduced-price lunch $(33.21 \%)$, and neither subsidy (32.83\%). 


\subsubsection{Recruitment and data quality checks.}

Per the institutional review board that approved this project, adolescents participated in the study after parental consent was given. Thus, Qualtrics first made the survey available to participants who had children in the required age range. Having initiated the survey, parent participants completed screening questions, were informed about the nature of this study, and were then asked to invite an adolescent child to complete the questionnaire.

We employed three data quality checks to ensure that adolescents were participating in the study, and to ascertain that participants were paying attention to the questions. First, we compared aggregate parent and adolescent responses on a key question to which, according to previous research, parents and teens respond divergently (Vaughan, Feinstein, Peugh, \& ShandLubbers, 2011). Using a four-point scale (1 = "very good" to 4 = "not good at all"), parent respondents indicated that they were better at listening to their children $(M=3.51, S D=.60)$ than how adolescent respondents rated their parents' listening to them $(M=3.36, S D=.62)(t=$ 2.92, $p=.004)$. We also asked the adolescent respondents five teen culture questions (e.g., "How many seconds is a Vine video?"). As expected, adolescent respondents knew the answers to most of these questions. The average score was 3.45 out of 5 , and $79.02 \%$ of the respondents answered three or more of the questions correctly. Omitting respondents who did not score well on these questions did not substantively change any of the study's results.

Three attention checks were used in the questionnaire (i.e., items directing participants to select a specific response), to target participants who were not reading the items. Failing one of the attention checks terminated the questionnaire and a new participant was recruited into the study. Of the 276 participants who passed the three attention checks, nine participants were eliminated because they provided bogus responses on open-ended questions. Two other 
participants were eliminated because they did not complete most of the questionnaire items, resulting in the final dataset of 265 participants.

\subsection{Procedure}

After consenting to participate, adolescent participants were informed that they would be testing a new social media platform dubbed "Mee." They were asked to choose content to display in their Mee profiles that would represent them accurately. Participants were presented with seven screens, each displaying six items from one content category. They were asked to select one item from each content category to display in their social media profiles. The seven content categories were female photos, male photos, song lyrics, TV channels, clothing brands, video games, and magazines. Items within each category varied in sexual intensity (i.e., low, medium, high). Pretesting procedures for these items are described below. To prevent order effects, the content categories and individual items within each category were displayed in random order. Figure 3 illustrates the selection screen for the TV channel category. 


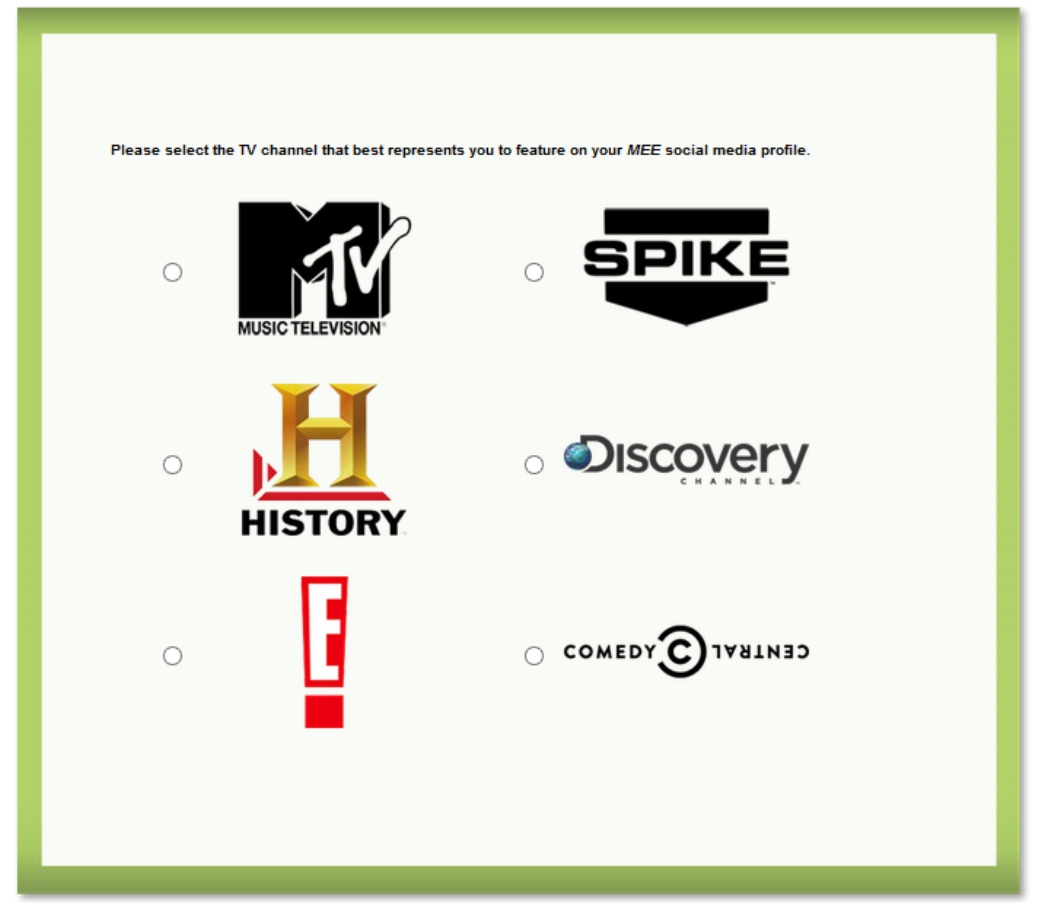

Figure 3

Example of a content selection screen for the TV channel category. Based on pretest scores, items represented three levels of sexual intensity: low (1; History, Discovery), medium (2; MTV, Spike), and high (3; E!, Comedy Central). Items were displayed in random order.

After selecting one item from each of the seven content categories and after indicating whether they preferred the male or female photo to appear as the cover photo in the profile (i.e., largest photo), participants viewed the complete social media profile containing the seven content items they selected. After viewing the profile, participants answered questions about their demographics, media use, personality traits, sexual experience, and their sexual selfconcept. The questionnaire's final screen debriefed respondents on the study's goals and procedures and explained the fictitious nature of the social media profile. 


\section{Table 1}

Descriptive statistics of the study main variables.

\begin{tabular}{|c|c|c|c|c|}
\hline & \multicolumn{3}{|c|}{ Original } & \multirow{2}{*}{ Centered range } \\
\hline & Range & $M$ & $S D$ & \\
\hline Sexual intensity & $1-2.86$ & 1.76 & .38 & $-2.00-2.88$ \\
\hline Sexual self-concept & $1.27-5.72$ & 3.30 & .81 & $-2.55-3.03$ \\
\hline Sexual media diet & $0-7$ & 2.03 & 1.43 & $-1.42-3.52$ \\
\hline Extraversion & $1-6$ & 4.45 & .91 & $-3.83-1.70$ \\
\hline Gender (female) & $0-1$ & .55 & .50 & - \\
\hline Age & $13-15$ & 13.89 & .73 & - \\
\hline Ethnicity (white) & $0-1$ & .69 & .46 & - \\
\hline
\end{tabular}

\subsection{Measures}

Table 1 presents the descriptive statistics for all variables used in the analyses. Nondemographic independent and dependent variables were standardized such that each distribution had mean of zero and a standard deviation of one.

\subsubsection{Sexual intensity}

The intensity level of respondents' online sexual self-presentations was measured using the seven content items that respondents selected for inclusion in their personal social media profiles. The sets of items from which participants selected profile content were first pretested with a convenience sample of 354 undergraduate students. Between 20 and 30 items per category 
were identified originally from teen trend websites and teen informants. Each pretest participant rated four items from each content category. Participants rated the sexual intensity of each item using four six-point semantic differential scales, each anchored by a pair of sex-related antonyms (e.g., "sexual" ... "nonsexual;" "G-rated" ... "X-rated"). The order in which these scales were displayed was randomized. Scores from the seven scales were summed, with each item receiving an overall sexiness score. Within each content category, items were grouped into sexual intensity levels based on statistically significant differences and non-significant differences in their individual intensity scores.

The six items in each content category that were presented to the adolescent participants were selected from the pretest intensity groupings to represent three significantly discrete levels of sexual intensity $(1=$ low, $2=$ medium, 3 = high $)$. To expand the participants' options, two items per category represented each of the three intensity levels.

Each participant's sexual intensity score was the average of the scores for the seven items the participant selected for his/her social media profile $(\alpha=.79)$. Figure 4 shows two profile examples that communicate different levels of sexual intensity. 

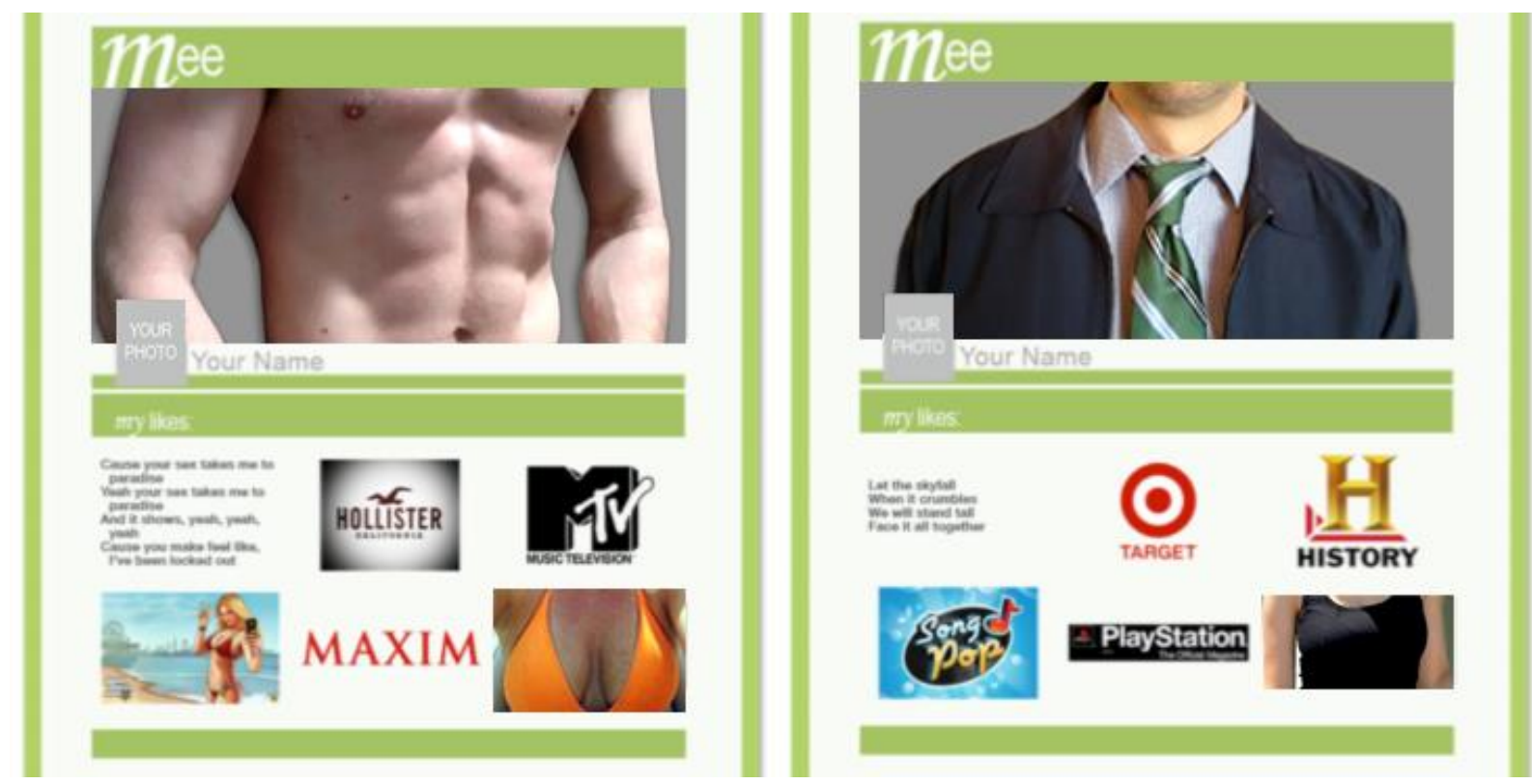

Figure 4

Two possible social media profiles, each constructed from seven content items. Left profile communicates a high level of sexual intensity (score: 3 ); right profile communicates a low level of sexual intensity (score: 1).

\subsubsection{Sexual self-concept}

Respondents completed Winter's (1988) sexual self-concept scale, which consisted of 12 questions assessing self-perceived sexual readiness and sexual self-efficacy. All participants answered the nine scale questions that did not presume prior sexual activity (e.g., "I consider myself emotionally ready for a sexual relationship"). Based on a sexual experience screening item ("Have you ever had sexual intercourse"), respondents who answered yes or no were routed accordingly to three additional scale questions about having sex, which were expressed in either a present or conditional form (i.e., "I think having sex gives me a bad reputation," or "I think having sex would give me a bad reputation"). Respondents answered using a six-point scale (i.e., 
$1=$ "Strongly disagree" to $6=$ "Strongly agree"). One item was omitted because of a low factor

loading. The resulting 11-item scale had appropriate internal consistency $(\alpha=.78)$.

\subsubsection{Sexual media diet}

Respondents indicated how many days in the previous week, at any point during the day $(0=$ "none" to $7=$ "every day") they watched, listened to, or looked at nine media categories that are known to include substantial sexual content (Pardun et al., 2005), that is prime time television, music videos, premium cable channels (i.e., HBO, Showtime, Cinemax, Starz), Rrated movies, adult or X-rated movies or clips (showing naked people or people engaged in sexual acts), sexually playful or suggestive text/phone messages (i.e., "sexting”), video games rated $\mathrm{M}$ for mature, and rap and/or hip-hop music. Participants' mean frequency of the numbers of days in the previous week they used all of these media was the final measure of sexual media $\operatorname{diet}(\alpha=.86)$.

\subsubsection{Extraversion}

Respondents rated their level of extraversion using the introversion-extraversion component of Goldberg's (1992) "Big Five” inventory. Respondents were asked to select values that best described them on seven six-point semantic differential scales, each anchored by a pair of adjectives (e.g., "unenergetic ... energetic," "bold ... timid"). The extraversion scale had good internal consistency $(\alpha=.87)$.

\subsubsection{Control variables}

Three demographic variables were used as controls: gender $(0=$ male, $1=$ female $)$, age (13-15), and race/ethnicity $(0=$ non-white, $1=$ white $)$, because these variables have been shown consistently to correlate with online sexual communication (Baumgartner et al., 2014; Dake et al., 2012). 
To account for the potential parental influence while the adolescent participants answered sensitive questions, we asked participants if their parents were in the room. We did not account for this in the final statistical models because the key coefficients did not change substantively when we excluded these participants from the analysis or accounted for them with a dummy variable.

\section{Results}

The proposed mediated moderation model (Figure 2) was tested by estimating its constituent path models, as recommended by Hayes (2013). See Table 2 for all corresponding coefficients. Hypotheses proposing direct and mediated relationships are discussed first, followed by the hypotheses on the proposed moderators.

\subsection{H1: Sexual Self-Concept to Sexual Intensity in Online Self-Presentations}

The regression model addressing $\mathrm{H} 1$ and predicting sexual intensity from the sexual selfconcept and the three control variables was estimated first (Table 2, Model 1). The sexual selfconcept coefficient was positive and statistically significant, indicating that as sexual selfconcept increased, the intensity of sexual self-presentation also increased, as indicated by the sexual intensity of the items adolescents chose for self-presentation in the online profile. H1 was thus supported. Additionally, the gender coefficient was negative and statistically significant, showing that male respondents tended to present themselves with greater sexual intensity than female respondents. The age and ethnicity coefficients were not statistically significant and unrelated to sexual intensity.

\subsection{H2: Sexual Self-Concept to Sexual Media Diet}

Model 2 in Table 2 presents the regression model predicting sexual media diet from the sexual self-concept and the control variables (H3). The sexual self-concept coefficient was positive and statistically significant, indicating that as the sexual self-concept increased, sexual 
media diet also increased. H3 was thus supported. The ethnicity coefficient was negative and statistically significant, indicating that non-white respondents tended to have a more sexual media diet than white respondents. The age and gender coefficients were not statistically significant in this model.

\subsection{H3: Sexual Media Diet to Sexual Intensity in Online Self-Presentations}

Model 3 in Table 2 presents the regression model predicting sexual intensity of selfpresentation from the sexual self-concept, sexual media diet, and control variables. The sexual self-concept and sexual media diet coefficients were positive and statistically significant, indicating that sexual intensity of self-presentation increased as a function of both the sexual self-concept and, as predicted in $\mathrm{H} 4$, sexual media diet.

\subsection{H4: Sexual Media Diet as a Mediator}

The results of Models 2 and 3 together suggest that sexual media diet partially mediated the effect of sexual self-concept on sexual intensity. The direct effect coefficient of sexual selfconcept on sexual intensity was .19 and statistically significant $(p=.002)$. The indirect effect coefficient through sexual media diet was .13 and also statistically significant, according to PROCESS-estimated bootstrap confidence intervals using 1,000 bootstrap samples (CI: .08-.19). H5 was thus supported. 


\section{Table 2}

Regression models predicting sexual intensity (Models 1, 3 and 4), and sexual media diet (Model 2) $(\mathrm{N}=265)$.

$B \quad S E \quad p$

Model 1: Predicting sexual intensity

\begin{tabular}{llrrr} 
& Sexual self-concept & .32 & .06 & $<.001$ \\
Gender & -.28 & .12 & .018 \\
& Age & .12 & .08 & .146 \\
& Ethnicity & -.22 & .13 & .078 \\
& Intercept & -1.30 & 1.11 & .244 \\
& $R^{2}$ & .17 & & \\
\hline
\end{tabular}

Model 2: Predicting sexual media diet

Sexual self-concept

$.41 \quad .06<.001$

Gender

$.04 \quad .12 \quad .762$

Age

$\begin{array}{lll}-.03 & .08 \quad .672\end{array}$

ssc

Ethnicity

$-.34$

$.12 \quad .007$

Intercept

$\begin{array}{lll}.69 & 1.11 \quad .534\end{array}$

$R^{2}$

.20

Model 3: Predicting sexual intensity

Sexual self-concept

\begin{tabular}{llrrr} 
& Sexual self-concept & .19 & .06 & .002 \\
Sexual media diet & .32 & .06 & $<.001$ \\
Gender & -.29 & .11 & .010 \\
Age & .13 & .08 & .095 \\
Est & Ethnicity & -.11 & .12 & .348 \\
Intercept & -1.53 & 1.06 & .151 \\
\hline
\end{tabular}



$R^{2}$
.26

Model 4: Predicting sexual intensity

\begin{tabular}{|c|c|c|c|c|}
\hline & Sexual self-concept & .17 & .06 & .009 \\
\hline & Sexual media diet & .31 & .06 & $<.001$ \\
\hline & Extraversion & .06 & .06 & .285 \\
\hline & Sexual self-concept $\times$ Extraversion & .16 & .06 & .007 \\
\hline & Sexual media diet $\times$ Extraversion & -.15 & .06 & .007 \\
\hline & Gender & -.24 & .11 & .029 \\
\hline & Age & .13 & .08 & .084 \\
\hline & Ethnicity & -.10 & .12 & .423 \\
\hline & Intercept & -1.62 & 1.05 & .125 \\
\hline & $R^{2}$ & .29 & & \\
\hline
\end{tabular}

Note: $\mathrm{SSC}=$ Sexual self-concept $; \mathrm{SI}=$ Sexual intensity; $\mathrm{SMD}=$ Sexual media diet $; \mathrm{E}=$ Extraversion.

\subsection{H5 and H6: Extraversion as a Moderator}

Finally, the moderating role of extraversion was examined (as proposed in $\mathrm{H} 2$ and $\mathrm{H} 6$ ). Model 4 in Table 2 presents the regression model predicting sexual intensity from the sexual self-concept, sexual media diet, extraversion, the two interaction terms, and control variables. Both of the interaction coefficients were statistically significant and are illustrated in Figure 5 (sexual self-concept $\times$ extraversion) and Figure 6 (sexual media diet $\times$ extraversion).

According to Figure 5, the sexual intensity scores of below-average extraverts (left of the broken vertical line; $.50 S D$ below $M$ and lower) did not differ as a function of the sexual selfconcept. In other words, the intensity of an introvert's sexual self-presentation did not increase or 
weaken based on his/her sexual self-concept. Among above-average extraverts (right of the broken vertical line), however, those with a high sexual self-concept (red line) tended to present themselves sexually with greater intensity than those with a low sexual self-concept (blue line). Thus, as predicted in $\mathrm{H} 2$, extraversion intensified the sexual self-presentation of those with a high sexual self-concept. H2 was thus supported.

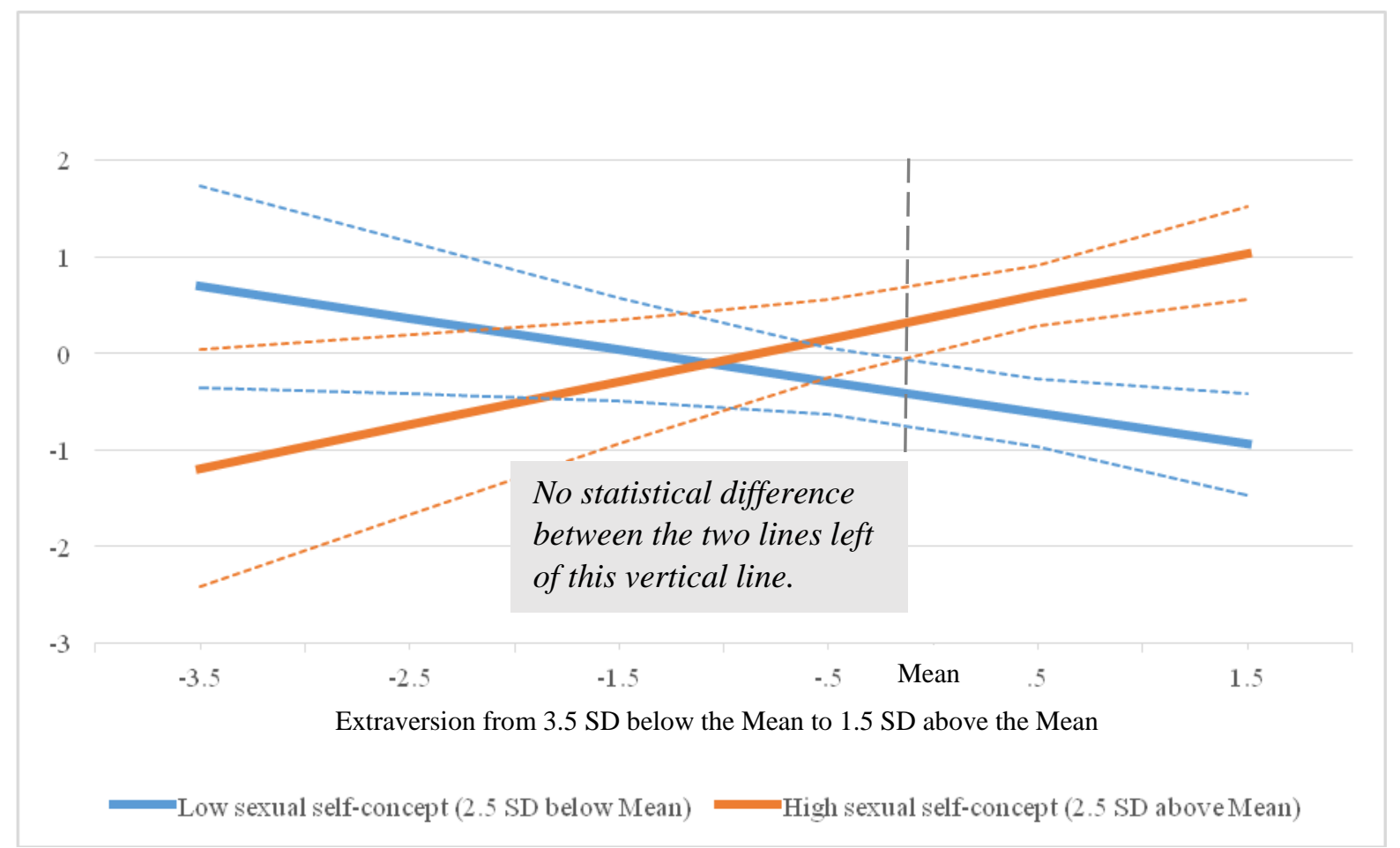

Figure 5

Sexual intensity of self-presentation as a function of the sexual self-concept and extraversion.

According to Figure 6, below-average, average, and slightly above-average extraverts (up to $.75 S D$ above $M$; left of the broken line) who had a highly sexual media diet (red line) tended to present themselves sexually with greater intensity than equally extraverted individuals without a sexy media diet (blue line). Above-average extraversion erased this difference. The nature of 
this interaction was somewhat counterintuitive, then, and did not fully support H6. Whereas we expected that extraversion would amplify sexual intensity especially among those who consumed higher quantities of sexual media, sexual media diet in this model did not predict sexual intensity among high extraverts. Instead, high extraverts presented themselves with equal sexual intensity regardless of how much sexual media they consumed. H6 was not supported.

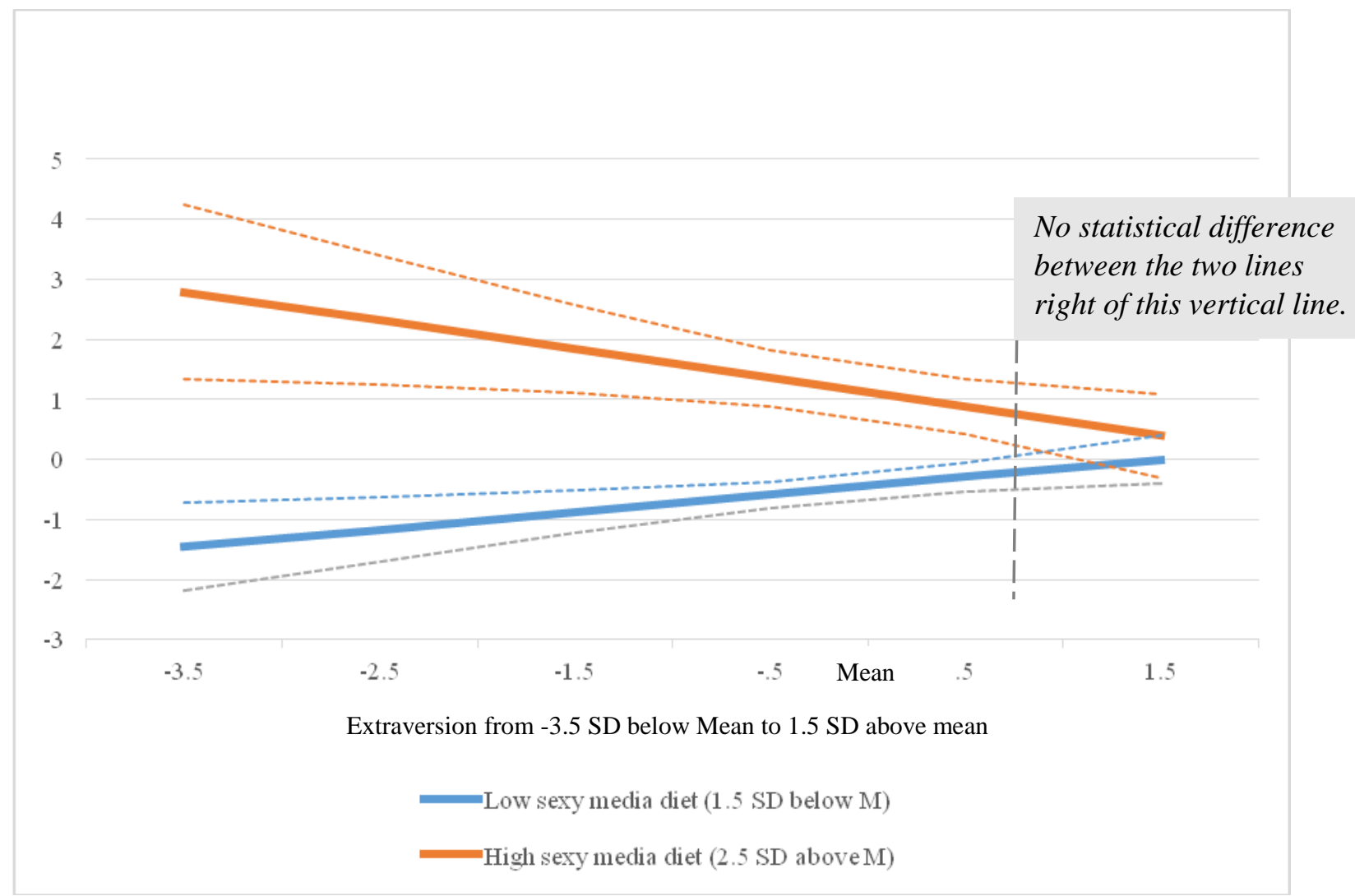

Figure 6

Sexual intensity of self-presentation as a function of the sexual media diet and extraversion.

In sum, the effect of the sexual self-concept on the intensity of sexual self-presentation was mediated partially through sexual media diet. Both the direct and indirect effects were moderated by extraversion. High extraversion intensified the sexual content of those with a high 
sexual self-concept. Sexual media diet, meanwhile, intensified the sexual content of low-toabove-average extraverts but had no effect on the sexual intensity of high extraverts.

\section{Discussion}

Armed with their smart phones, tablets, and laptops, today's adolescents create and distribute a vast quantity of digital media content, some of which may be sexual. While much of the previous research in this area has focused on sexually explicit text and photos (e.g., Mitchell et al., 2012; Temple \& Choi, 2014), this study examined adolescents' sexual self-presentations more broadly. Because young people can self-present sexually without showing nudity or nearnudity, we asked adolescent participants to select seven non-nude media items with which they wanted to present themselves in a social media profile. While not containing nudity, the items did vary in the level of "sexiness" (e.g., Hollister being perceived as a "sexier" brand than Old Navy). Based on the items participants chose, we computed the average level of sexual intensity with which the participants wanted to represent themselves.

Guided by the Media Practice Model (Shafer et al., 2013), our study locates sexual selfpresentation as a product of identity, sexual media use, and personality. This stands in contrast to prior research, which focused on the links between sexual self-presentation (i.e., sexting) and discrete personal and relational correlates like gender, personality traits, and relationship status (e.g., Delevi \& Weisskirch, 2013; Drouin \& Tobin, 2014; Mitchell et al., 2012). This study illustrates that the sexual intensity with which adolescents present themselves digitally is shaped by their sexual self-concepts, that is, how sexually mature and efficacious they perceive themselves. The study also finds that as a fundamental element of personality, extraversion works in concert with the sexual self-concept, differentiating the level of sexual intensity with which more sexual adolescents, versus less sexual adolescents, present themselves. Finally, the study shows that the volume of sexual media that adolescents consume influences the intensity of 
their sexual self-presentations. Adolescents who use sexual media more frequently present themselves with greater sexual intensity than their peers who use sexual media less frequently. This difference is especially evident among introverts.

The study's limitations include its cross-sectional design, the online character of its questionnaire and participant panel, and the one-dimensional nature of its sexual self-concept measure. First, sexual self-presentation constitutes one link in a reciprocal chain of media and sexual cognitions and behaviors (Shafer et al., 2013; Slater, 2007). This study, in contrast, measured adolescents' sexual self-concepts, sexual media diet, and sexual intensity in a crosssectional survey and was thus unable to assert causality and account for the dynamic and reciprocal relationships between these variables. Second, the study used an online participant panel, which while not uncommon in communication research (e.g., Morey, Eveland, \& Hutchens, 2012), limits the researcher's control over the environments in which participants complete the online questionnaire. Although we eliminated participants who did not pay attention to the questions and accounted for the potential influence of a parent's presence, other external factors for which we were unable to account may have contributed to unexplained error in our statistical models. Finally, although some researchers have conceptualized sexual selfconcept as a multi-dimensional construct (O’Sullivan, Meyer-Bahlburg, \& McKeague, 2006; Snell, 1998), this study employed a one-dimensional sexual self-concept scale to streamline the questionnaire and minimize participant fatigue. A multi-dimensional measure would have allowed greater nuance with which to understand the role of sexual self-concept elements in sexual self-presentation.

This study's findings can inform future research and theory development in the areas of sexual media uses and effects and sexual self-presentation. Most important, the study understands sexual self-presentation within the conventional context of adolescents' media uses 
and sexual behaviors. This suggests that sexual self-presentation can be a media effect, that is, an outcome of consuming sexual media, which thus places sexual self-presentation, including sexting, alongside other effects of adolescent sexual media consumption, such kissing, oral sex, sexual debut, and unplanned pregnancy (Brown et al., 2006; Chandra et al., 2008; Martino, Collins, Kanouse, Elliott, \& Berry, 2005). Sexual self-presentation can also then fit within a research literature that developed largely before young people became prolific producers and distributors of digital content but which can greatly inform research and understanding of these behaviors (for a review, see Wright, 2011).

The literature on sexual media effects provides rich socio-psychological frameworks within which to understand sexual self-presentation. Like other sexual media effects, sexual selfpresentation occurs in the context of sexual norms that adolescents inherit from their families and peer groups (Browning, Leventhal, \& Brooks-Gunn, 2004; O’Donnell, Myint-U, O’Donnell, \& Stueve, 2003). Adolescents' sexual norms shape the ways in which they process the sexual media they consume (Martino, et al., 2005) and likely also whether they produce sexual media of their own. The processing mechanisms that mediate the link between sexual media exposure and sexual behaviors, such as sexual script acquisition and enactment (Shafer et al., 2013; Wright, 2011), similarly can influence adolescents' propensity for producing sexual content. How much adolescents pay attention to sexual media and identify with the individuals featured in these media, for instance, determines the extent to which they cognitively assimilate the media's sexual scripts (Jeong, Hwang, \& Fishbein, 2010; Ward \& Friedman, 2006), which they may eventually enact in their own media. The sexual media that adolescents consume also may communicate the consequences of sexual behaviors and stimulate a sense of self-efficacy for enacting sexual scripts (Eyal \& Kunkel, 2008; Martino et al., 2005), which may include the 
creation of sexual self-presentations. In all, the literature on sexual media effects contains developed conceptual frameworks within which to better understand sexual self-presentation.

The nature of a self-presentation, in general, results from an interplay between audience characteristics, the context of the presentation, and presenter characteristics (Schlenker, 2005; Shafer et al., 2013). Future research may build on this study's findings by accounting for audience and platform attributes, in addition to the presenter characteristics examined here. The intended audience of the self-presentation is a unique component of sexual self-presentation, and one that this study did not assess. Adolescents' relationships with the intended recipients of their sexual self-presentations, and their goals for these relationships, likely determine the sexual intensity of the self-presentations (Shafer et al., 2013). Related to this are the digital platforms adolescents use and the agreed-upon functions of these platforms. This study asked participants to construct a profile in a fictitious social media platform but actual social media differ in terms of the functions their users ascribe to them, and the extent to which they understand them as potential venues for sexual self-presentation. For instance, whereas today's adolescents may present themselves with no or little sexual intensity on Facebook because Facebook's perceived function is to connect with parents and grandparents, adolescents may find Snapchat to be a conducive medium for sexually intense self-presentations because of Snapchat's younger user base and the self-destructing nature of Snapchat messages (Utz, Muscanell, \& Khalid, 2015).

Finally, this study leaves open the question of outcomes of sexual self-presentation. In contrast to prior work (e.g., Mitchell et al., 2012), this study demonstrates the utility of measuring sexual self-presentation without the assumption of explicit nudity. Young people's digital self-presentations fall on a continuum of sexual intensity and future studies may examine the consequences of self-presentation across the range of this continuum. Unlike conventional media effects research, research on the consequences of digital self-presentations may consider 
not only how the self-presentations affect their recipients but also how creating and distributing sexual media affects the individuals who create and distribute the sexual messages. Articulating a message for a media audience can be transformative for the message sender (Pingree, 2014). Creating sexual self-presentations may increase adolescents' sense of sexual self-efficacy, for instance, and solidify their sexual self-concepts. Behaviorally, sexual self-presentations may hasten the onset of adolescents' sexual activity (Temple \& Choi, 2014). Receiving sexual selfpresentations from peers also may influence adolescents' sexual development. The greater the similarity between individuals modeling sexual behaviors in the media and the adolescent recipients of these media, the greater the likelihood of these adolescents cognitively encoding and behaviorally enacting the sexual scripts communicated in the media (Wright, 2011). Adolescents' digital sexual self-presentations may have an outsized effect on their friends who receive these self-presentations. In all, the field is ripe for sustained research efforts to better understand the precursors and effects of adolescents' sexual communication behaviors in digital spaces.

\section{Conclusion}

Drawing on the precepts of the Media Practice Model, this study showed that the sexual intensity with which adolescents (13 to 15 years old) present themselves online depends on a combination of the adolescents' sexual self-concepts, sexual media diets, and extraversion. The study positioned sexual self-disclosure in digital spaces (e.g., sexting) as a form of a media effect, in line with other effects of consuming sexual media (Brown et al., 2006; Chandra et al., 2008; Martino et al., 2005). As such, the study suggests using established sexual media effects literature to understand better the inspiration that adolescents draw from sexual media for creating and distributing sexual media of their own, the mediating factors that attenuate these 
behaviors, and the implications that producing sexual media has for the teen producers and recipients of these media.

\section{References}

Amichai-Hamburger, Y., \& Vinitzky, G. (2010). Social network use and personality. Computers in Human Behavior, 26(6), 1289-1295. doi:10.1016/j.chb.2010.03.018

Archer, R. L. (1979). Role of personality and the social situation. In G. I. Chelune and Associates, Self-disclosure: Origins, patterns, and implications of openness in interpersonal relationships (pp. 28-58). San Francisco: Jossey-Bass.

Baumgartner, S. E., Sumter, S. R., Peter, J., Valkenburg, P. M., \& Livingstone, S. (2014). Does country context matter? Investigating the predictors of teen sexting across Europe. Computers in Human Behavior, 34, 157-164. doi:10.1016/j.chb.2014.01.041

Bleakley, A., Hennessy, M., \& Fishbein, M. (2011). A model of adolescents's seeking of sexual content in in their media choices. The Journal of Sex Research, 48(4), 309-315. doi:10.1080/00224499.2010.497985

Bobkowski, P. S., Brown, J. D., \& Neffa, D. R. (2012). “Hit me up and we can get down:” U.S. youths' risk behaviors and sexual self-disclosure in MySpace profiles. Journal of Children and Media, 6(1), 119-134. doi:10.1080/17482798.2011.633412

Breakwell, G. M. \& Millward, L. J. (1997). Sexual self-concept and sexual risk-taking. Journal of Adolescence, 20(1), 29-41. doi:10.1006/jado.1996.0062

Brown. J. D., L'Engle, K. L., Pardun, C. J., Guo, G., Kenneavy, K., \& Jackson, C. (2006). Sexy media matter: Exposure to sexual content in music, movies, television, and magazines predicts black and white adolescents' sexual behavior. Pediatrics, 117(4), 1018-1027. doi:10.1542/peds.2005-1406 
Browning, C. R., Leventhal, T., \& Brooks-Gunn, J. (2004). Neighborhood context and racial differences in early adolescent sexual activity. Demography, 41(4), 697-720. doi:10.1353/dem.2004.0029

Carpentier, F. R. D. (2008). Applicability of the informational utility model for radio news. Journalism \& Mass Communication Quarterly, 85(3), 577-590. doi:10.1177/107769900808500306

Chandra, A., Martino, S. C., Collins, R. L., Elliot, M. N., Berry, S. H., Kanouse, D. E., \& Miu, A. (2008). Does watching sex on television predict teen pregnancy? Findings from a national longitudinal survey of youth. Pediatrics, 122(5), 1047-1054. doi:10.1542/peds.2007-3066

Cozby, P. C. (1973). Self-disclosure: A literature review. Psychological Bulletin, 79(2), 73-91. doi:10.1037/h0033950

Crimmins, D. M., \& Seigfried-Spellar, K. C. (2014). Peer attachment, sexual experiences, and risky online behaviors as predictors of sexting behaviors among undergraduate students. Computers in Human Behavior, 32, 268-275. doi:10.1016/j.chb.2013.12.012

Dake, J. A., Price, J. H., Maziarz, , L., \& Ward, B. (2012). Prevalence and correlates of sexting behavior in adolescents. American Journal of Sexuality Education, 7(1), 1-15. doi:10.1080/15546128.2012.650959

Delevi, R., \& Weisskirch, R. S. (2013). Personality factors as predictors of sexting. Computers in Human Behavior, 29, 2589-2594. doi:10.1016/j.chb.2013.06.003

Desjarlais, M., \& Willoughby, T. (2010). A longitudinal study of the relation between adolescent boys and girls' computer use with friends and friendship quality: Support for the social compensation of the rich-get-richer hypothesis? Computers in Human Behavior, 26(5), 896-905. doi:10.1016/j.chb.2010.02.004 
Dir, A. L., Coskunpinar, A., Steiner, J. L., \& Cyders, M. A. (2013). Understanding differences in sexting behaviors across gender, relationship status, and sexual identity, and the role of expectancies in sexting. Cyberpsychology, Behavior, and Social Networking, 16(8), 568574. doi:10.1089/cyber.2012.0545.

Doornwaard, S. M., van den Eijnden, R. J. J M., Overbeek, G., \& ter Bogt, T. F. M. (2015). Differential developmental profiles of adolescents using sexually explicit Internet material. Journal of Sex Research, 52(3), 269-281. doi:10.1080/00224499.2013.866195

Drouin, M., \& Tobin, E. (2014). Unwanted but consensual sexting among young adults: Relations with attachment and sexual motivations. Computers in Human Behavior, 31, 412-418. doi:10.1016/j.chb.2013.11.001

Drouin, M., Vogel, K. N., Surbey, A., \& Stills, J. R. (2013). Let's talk about sexting, baby: Computer-mediated sexual behaviors among young adults. Computers in Human Behavior, 29(5), A25-A30. doi:10.1016/j.chb.2012.12.030

Eyal, K., \& Kunkel, D. (2008). The effects of sex in television drama shows on emerging adults' sexual attitudes and moral judgments. Journal of Broadcasting \& Electronic Media, 52(2), 161-181. doi:10.1080/08838150801991757

Goldberg, L. R. (1992). Development of markers for the Big-Five factor structure. Psychological Assessment, 4(1), 26-42. doi:10.1037//1040-3590.4.1.26

Gordon-Messer, D., Bauermeister, J. A., Grodzinski, A., \& Zimmerman, M. (2013). Sexting among young adults. Journal of Adolescent Health, 52(3), 301-306. doi:10.1016/j.jadohealth.2012.05.013

Hawk, S. T., Vanwesenbeeck, I., de Graaf, H., \& Bakker, F. (2006). Adolescents' contact with sexuality in mainstream media: A selection-based perspective. The Journal of Sex Research, 43(4), 352-363. doi: 10.1080/00224490609552334 
Hayes, A. F. (2013). Introduction to mediation, moderation, and conditional process analysis: A regression-based approach. New York, NY: Guilford.

Ho, J. Y. C., \& Dempsey, M. (2010). Viral marketing: Motivations to forward online content. Journal of Business Research, 63, 1000-1006. doi: 10.1016/j.jbusres.2008.08.010

Impett, E. A., \& Tolman, D. L. (2006). Late adolescent girls' sexual experiences and sexual satisfaction. Journal of Adolescent Research, 21(6), 628-646. doi:10.1177/0743558406293964

Jeong, S. H., Hwang, Y., \& Fishbein, M. (2010). Effects of exposure to sexual content in the media on adolescent sexual behaviors: The Moderating Role of Multitasking with Media. Media Psychology, 13(3), 222-242. doi:10.1080/15213269.2010.502872

Jones, J. J., Settle, J. E., Bond, R. M., Farris, C. J., Marlow, C., \& Fowler, J. H. (2013). Inferring tie strength from online directed behavior, PLOS ONE, 8(1), 1-6. doi:10.1371/journal.pone. 0052168

Klettke, B., Hallford, D. J., \& Mellor, D. J. (2014). Sexting prevalence and correlates: A systematic literature review. Clinical Psychology Review, 34(1), 44-53. doi:10.1016/j.cpr.2013.10.007

Knobloch, S., Carpentier, F. D., \& Zillmann, D. (2003). Effects of salience dimensions of informational utility on selective exposure to online news. Journalism \& Mass Communication Quarterly, 80(1), 91-108. doi:10.1177/107769900308000107

Lee, E., Ahn, J., \& Kim, Y. J. (2014). Personality traits and self-presentation at Facebook. Personality and Individual Differences, 69, 162-167. doi:10.1016/j.paid.2014.05.020

Macafee, T. (2013). Some of these things are not like the others: Examining motivations and political predispositions among political Facebook activity. Computers in Human Behavior, 29(6), 2766-2775. doi: 10.1016/j.chb.2013.07.019 
Madden, M. (2013, August 15). Commentary: Teens haven't abandoned Facebook (yet).

Retrieved from the Pew Internet and American Life Project website: http://pewinternet.org/2013/08/15/teens-havent-abandoned-facebook-yet/

Madden, M., Lenhart, A., Duggan, M., Cortesi, S., \& Gasser, U. (2013). Teens and technology 2013. Retrieved from the Pew Internet and American Life Project website: http://www.pewinternet.org/files/old-media/Files/Reports/2013/ PIP_TeensandTechnology2013.pdf

Madden, M., Lenhart, A., Cortesi, S., \& Gasser, U. (2013). Teens and mobile apps privacy. Retrieved from the Pew Internet and American Life Project website: http://www.pewinternet.org/2013/08/22/teens-and-mobile-apps-privacy/

Madden, M. Lenhart, A., Cortesi, S., Gasser, U., Duggan, M., Smith, A., \& Beaton, M. (2013). Teens, social media, and privacy. Retrieved from the Pew Internet and American Life Project website: http://www.pewinternet.org/2013/05/21/teens-social-media-and-privacy/

Martino, S., Collins, R., Kanouse, D., Elliott, M., \& Berry, S. (2005). Social cognitive processes mediating the relationship between exposure to television's sexual content and adolescents' sexual behavior. Journal of Personality \& Social Psychology, 89(6), 914 924. doi:10.1037/0022-3514.89.6.914

McCrae, R. R., \& John, O. P. (1992). An introduction to the Five-Factor Model and its applications. Journal of Personality, 60, 175-215. doi:0.1111/j.14676494.1992.tb00970.x

Michikyan, M., Subrahmanyam, K., \& Dennis, J. (2014). Can you tell who I am? Neuroticism, extraversion, and online self-presentation among young adults. Computers in Human Behavior, 33, 179-183. doi:10.1016/j.chb.2014.01.010 
Mitchell, K. J., Finkelhor, D., Jones, L. M., \& Wolak, J. (2012). Prevalence and characteristics of youth sexting: A national study. Pediatrics, 129(1), 13-20. doi:10.1542/peds.2011-1730

Morey, A. C., Eveland, W. P., \& Hutchens, M. J. (2012). The "who" matters: Types of interpersonal relationships and avoidance of political disagreement. Political Communication, 29(1), 86-103. doi:10.1080/10584609.2011.641070

O’Donnell, L., Myint-U, A., O’Donnell, C. R., \& Stueve, A. (2003). Long-term influence of sexual norms and attitudes on timing of sexual initiation among urban minority youth. Journal of School Health, 73(2), 68-75. doi:10.1111/j.1746-1561.2003.tb03575.x

O’Sullivan, L. F., Meyer-Bahlburg, H. F. L., \& McKeague, I. W. (2006). The development of the sexual self-concept inventory for early adolescent girls. Psychology of Women Quarterly, 30(2), 139-149. doi:10.1111/j.1471-6402.2006.00277.x

Pardun, C. J., L'Engle, K. L., \& Brown, J. D. (2005). Linking Exposure to Outcomes: Early Adolescents' Consumption of Sexual Content in Six Media. Mass Communication and Society, 8(2), 75-91. doi:10.1207/s15327825mcs0802_1

Peter, J., \& Valkenburg, P. M. (2006). Adolescents' exposure to sexually explicit material on the Internet. Communication Research, 33(2), 178-204. doi:10.1177/0093650205285369

Peter, J., Valkenburg, P. M., \& Schouten, A. P. (2005). Developing a model of adolescent friendship formation on the Internet. CyberPsychology \& Behavior, 8(5), 423-430. doi: $10.1089 / \mathrm{cpb} .2005 .8 .423$

Pingree, R. J. (2014). Effects of online political messages on their senders: Conceptual tools and research directions. In H. Gil de Zuniga (Ed.), New Agendas in Communication: New Technologies \& Civic Engagement. New York: Routledge. 
Reich, S. M., Subrahmanyam, K., \& Espinoza, G. (2012). Friending, IMing, and hanging out face-to-face: Overlap in adolescents' online and offline social networks. Developmental Psychology, 48, 356-368. doi: 10.1037/a0026980

Rice, E., Rhoades, H., Winetrobe, H., Sanchez, M., Montoya, J., Plant, A., \& Kordic, T. (2012). Sexually explicit cell phone messaging associated with sexual risk among adolescents. Pediatrics, 130(4), 667-673. doi:10.1542/peds.2012-0021

Ringrose, J. (2011). Are you sexy, flirty or a slut? Exploring "sexualization" and how teen girls perform/negotiate digital sexual identity on social networking sites. In R. Gill \& C. Scharff (Eds.), New femininities: Postfeminism, neoliberalism and subjectivity (pp. 99116). London, UK: Palgrave.

Rostosky, S. S., Dekhtyar, O., Cupp, P. K., \& Anderman, E. M. (2008). Sexual self-concept and sexual self-efficacy in adolescents: A possible clue to promoting sexual health? Journal of Sex Research, 45(3), 277-286. doi:10.1080/00224490802204480

Rothman, E., Kaczmarsky, C., Burke, N., Jansen, E., \& Baughman, A. (2014). “Without porn ... I wouldn't know half the things I know now": A qualitative study of pornography use among a sample of urban, low-inclome, black and Hispanic youth. Journal of Sex Research,52(7), 736-746. doi:10.1080/00224499.2014.960908

Ryan, T., \& Xenos, S. (2011).Who uses Facebook? An investigation into the relationship between the Big Five, shyness, narcissism, loneliness, and Facebook usage. Computers in Human Behavior, 27(5), 1658-1664. doi:10.1016/j.chb.2011.02.004

Schlenker, B. R. (2005). Self-presentation. In M.R. Leary \& J.P. Tangney (Eds.), Handbook of self and identity (pp. 492-518). New York: Guilford Press. 
Shafer, A., Bobkowski, P., \& Brown, J. D. (2013). Sexual media practice: How adolescents select, engage with, and are affected by sexual media. In K. E. Gill (ed.), The Oxford Handbook of Media Psychology (pp. 223-251). New York, NY: Oxford University Press.

Shah, D. V., \& Scheufele, D. A. (2006). Explicating opinion leadership: Nonpolitical dispositions, information, consumption, and civic participation. Political Communication, 23(1), 1-22. doi: 10.1080/10584600500476932

Slater, M. D. (2007). Reinforcing spirals: The mutual influence of media selectivity and media effects and their impact on individual behavior and social identity. Communication Theory, 17(3), 281-303. doi:10.1111/j.1468-2885.2007.00296.x

Smahel, D., \& Subrahmanyam, K. (2014). Adolescent sexuality on the Internet: A developmental perspective. In F. M. Saleh, A. Grudinskas, Jr., and A. Judge (Eds.), Adolescent sexual behavior in the digital age: Considerations for clinicians, legal professionals, and educators (pp. 69-88). New York, NY: Oxford University Press.

Snell, Jr., W. E. (1998). The multidimensional sexual self-concept questionnaire. In C. M. Davis, W. L. Yarber, R. Bausenman, G. Schreer, \& S. L. Davis (Eds.), Handbook of sexualityrelated measures (pp. 521-524). Newbury Park, CA: Sage.

Snell, W. E., Jr., Fisher T. D., \& Schuh, T. (1992). Reliability and validity of the sexuality scale: A measure of sexual-esteem, sexual-depression, and sexual-preoccupation. Journal of Sex Research, 29(2), 261-273. doi:10.1080/00224499209551646

Steele, J.R. (1999). Teenage sexuality and media practice: Factoring in the influences of family, friends, and school. The Journal of Sex Research, 36(4), 331-341. doi: $10.1080 / 00224499909552005$ 
Steele, J. R., \& Brown, J. D. (1995). Adolescent room culture: Studying media in the context of everyday life. Journal of Youth and Adolescence, 24(5), 551-576. doi:10.1007/bf01537056

Stokes, C. E. (2007). Representin’ in cyberspace: Sexual scripts, self-definition, and hip hop culture in Black American adolescent girls' home pages. Culture, Health \& Sexuality, 9(2), 169-184. doi:10.1080/13691050601017512

Temple, J. R., \& Choi, H. (2014). Longitudinal association between teen sexting and sexual behavior. Pediatrics, 134(5), e1287-e1292. doi:10.1542/peds.2014-1974

Temple, J. R., Paul, J. A., Berg, P., Le, V. D., McElhany, A., \& Temple, B. W. (2012). Teen sexting and its association with sexual behaviors. Archives of Pediatrics \& Adolescent Medicine, 166(9), 828-833. doi:10.1001/archpediatrics.2012.835

Utz, S., Muscanell, N., \& Khalid, C. (2015). Snapchat elicits more jealousy than Facebook: A comparison of Snapchat and Facebook use. Cyberpsychology, Behavior, and Social Networking, 18(3), 141-146. doi:10.1089/cyber.2014.0479

Valenzuela, S., Kim, Y., \& Gil de Zúñiga, H. (2012). Social networks that matter: Exploring the role of political discussion for online political participation. International Journal of Public Opinion Research, 24(2), 163-184. doi:10.1093/ijpor/edr037

Vaughan, R., Feinstein, E., Peugh, J., \& Shand-Lubbers, J. (2011). Same family, different planet: Comparing the views of teens and parents on their relationship and potential substance use. Presented at the Annual Conference of the American Association for Public Opinion Research.

Ward, L. M. (2003). Understanding the role of entertainment media in the sexual socialization of American youth: A review of empirical research. Developmental Review, 23, 347-388. doi:10.1016/S0273-2297(03)00013-3 
Ward, L. M., \& Friedman, K. (2006). Using TV as a guide: Associations between television viewing and adolescents' sexual attitudes and behavior. Journal of Research on Adolescence, 16(1), 133-156. doi:10.1111/j.1532-7795.2006.00125.x

Wilt, J., \& Revelle, W. (2009). Extraversion. In M. R. Leary \& R. H. Hoyle (Eds.), Handbook of individual differences in social behavior (pp. 27-45). New York: Guilford.

Winter, L. (1988). The role of sexual self-concept in the use of contraceptives. Family Planning Perspectives, 20, 123-127. doi:10.2307/2135700

Wright, P. (2011). Mass media effects on youth sexual behavior: Assessing the claim for causality. In C.T. Salmon (Ed.), Communication Yearbook (pp. 343-386). New York, NY: Routledge.

Wright, M. F., \& Li, Y. (2011). The associations between young adults' face-to-face prosocial behaviors and their online prosocial behaviors. Computers in Human Behavior 27(5), 19591962. doi:10.1016/j.chb.2011.04.019 